\title{
Validação de método para determinação de resíduos de agrotóxicos em tomate: uma experiência laboratorial
}

\author{
Method validation for determination of pesticide residues in tomatoes: a laboratorial experience
}

\author{
Maria Helena Wohlers Morelli CARDOSO ${ }^{1 \star}$, Adherlene Vieira GOUVÊA ${ }^{1}$, \\ Armi Wanderley da NÓBREGA ${ }^{1}$, Shirley de Mello Pereira ABRANTES ${ }^{1}$
}

\section{Resumo}

Um modelo de procedimento para validação de método de ensaio para determinação de cinco agrotóxicos $(\gamma-\mathrm{HCH}$, clorotalonil, fenitrotiona, clorpirifós e procimidona em matriz tomate) é demonstrado através da análise cromatográfica. A amostra processada é extraída com $30 \mathrm{~mL}$ de acetona e em seguida com $60 \mathrm{~mL}$ de uma mistura diclometano: éter de petróleo (1:1). O volume total é centrifugado e a alíquota orgânica é filtrada sob $\mathrm{Na}_{2} \mathrm{SO}_{4}$. Um mililitro de extrato orgânico é concentrado e dissolvido em um mililitro de iso-octano. Um microlitro do extrato é analisado no cromatógrafo a gás com detector por captura de elétrons - CG/DCE. Foram avaliados seletividade, linearidade, repetitividade, recuperação e limites de detecção e de quantificação. As recuperações obtidas variaram de 70 a $110 \%$, considerando-se os níveis de adição de agrotóxicos/amostra de 0,02 a 2,50 mg. $\mathrm{kg}^{-1}$. Os limites de detecção do método variaram de 0,004 a 0,006 mg.kg-1 e os de quantificação entre 0,014 e 0,020 mg.kg-1.

Palavras-chave: agrotóxicos; tomate; cromatografia em fase gasosa; validação.

\begin{abstract}
A validation procedure model of a multiresidue method is presented for chromatographic analyses of five pesticides residues $\gamma$-HCH, chlorothalonil, fenitrothion, chlorpyrifos and procymidone applied on tomatoes. The tomatoes were processed and extracted by acetone plus a mixture of dichloromethane:petroleum benzine (1:1). The volume was centrifuged and was then filtered under $\mathrm{Na}_{2} \mathrm{SO}_{4}$. One milliliter of organic extract was concentrated then diluted in isooctane and one microliter was analyzed in the gas chromatograph with electron capture detector - GC/ECD. The parameters evaluated were selectivity, linearity, repeatability, recovery, and limits of detection and quantification. The recovery ranged from 70 to $110 \%$ in the concentration range of 0.02 to $2.50 \mathrm{mg} \cdot \mathrm{kg}^{-1}$. The limits of detection ranged from 0.004 to $0.006 \mathrm{mg} \cdot \mathrm{kg}^{-1}$ and the limits of quantification were between 0.014 to $0.02 \mathrm{mg} \cdot \mathrm{kg}^{-1}$.

Keywords: pesticides; tomato; gas chromatography; validation.
\end{abstract}

\section{Introdução}

A cultura do tomateiro (Lycopersicon esculentum, Mill.) encontra-se bastante desenvolvida no Brasil e seu fruto está entre as hortaliças mais consumidas no mundo, estando globalmente enraizado no hábito alimentar. $\mathrm{O}$ tomate pode ser utilizado tanto para o consumo direto como legume de mesa, ou então aproveitado na indústria para o preparo de massas, sucos ou conservas (ABIA, 2007).

Os tomates estão classificados dentro do grupo de alto risco em relação à exposição aos agrotóxicos, por serem necessárias, grande número de práticas agronômicas para a sua produção. Desta forma, são os frutos que mais recebem pulverizações de agrotóxicos, gerando problemas de saúde pública, contaminação do meio ambiente principalmente do solo e da água, além de altas taxas residuais dessas substâncias nos frutos (MOREIRA, 1995; SILVA et al., 2005).

Segundo o Levantamento Sistemático da Produção Agrícola do IBGE (BRASIL, 2008), o Brasil produziu no ano de 2007, aproximadamente três milhões e trezentas toneladas do fruto para uma estimativa da safra de 2008 de três milhões e setecentas toneladas com cerca de 60 mil hectares de área plantada.

O LMR - limite máximo de resíduo de cada agrotóxico para determinado hortifrutigranjeiro é regulamentado no Brasil pela Agência Nacional de Vigilância Sanitária (ANVISA) (BRASIL, 2003a, b). No entanto, o LMR pode não ser o mesmo em outros países, ocasionando barreiras fitossanitárias e consequentemente perdas econômicas para o Brasil.

O desenvolvimento de métodos analíticos simples, eficientes e de baixo custo é essencial para a avaliação e monitoramento dessas substâncias em produtos hortifrutigranjeiros. Esses métodos normalmente envolvem três etapas: extração dos agrotóxicos dos alimentos, limpeza ou derivatização do extrato orgânico obtido e detecção seguida por quantificação da concentração. Cada etapa tem sua importância, entretanto, os resultados de análise gerados pelo laboratório é que sinalizam a tomada de decisão para rejeição ou não do produto analisado.

Recebido para publicação em 20/2/2008

Aceito para publicação em 6/1/2009 (003232)

Departamento de Química, Instituto Nacional de Controle de Qualidade em Saúde - INCQS, Fundação Oswaldo Cruz - FIOCRUZ, Av. Brasil, 4365, Manguinhos, CEP 21045-900, Rio de Janeiro - RJ, Brasil, E-mail: helenawohlers@hotmail.com

${ }^{*}$ A quem a correspondência deve ser enviada 
Uma das competências de um laboratório em produzir dados confiáveis é demonstrada pela validação do método de ensaio, cujo processo permite demonstrar que ele é adequado ao uso pretendido. Desse modo, a extensão do termo "validação de um método" é bastante ampla e apresentada com diversas definições na literatura além de quais parâmetros de desempenho devem ser avaliados (EURACHEM, 1998; GREEN, 1996; FAJGELJ; AMBRUS, 2000; HUBER, 1998; BRASIL, 2007; NATA, 1997; THOMPSON; ELLISON; WOOD, 2002, 1999) . Outra questão não harmonizada diz respeito aos critérios de aceitabilidade adotados para cada parâmetro avaliado (HILL; REYNOLDS, 1999).

Diante da diversidade de documentos existentes sobre o assunto em questão, o objetivo deste trabalho é apresentar um procedimento de validação aplicado a um método quantitativo para o ensaio de preparação e certificação de um material de referência a ser usado no controle de agrotóxicos em hortifrutigranjeiros (CARDOSO; ABRANTES; NÓBREGA, 2005), embasado em fundamentos teóricos. A discussão detalhada para aplicação dos parâmetros seguidos é encontrada na literatura, e não será apresentada neste trabalho (EURACHEM, 1998; GREEN, 1996; FAJGELJ; AMBRUS, 2000; HILL; REYNOLDS, 1999; HUBER, 1998; BRASIL, 2007; NATA, 1997; THOMPSON; ELLISON; WOOD, 2002; WOOD, 1999).

A aplicação foi demonstrada para cinco agrotóxicos, $\gamma-\mathrm{HCH}$ (organoclorado), clorotalonil (isoftalonitrila), procimidona (dicarboximida), fenitrotiona e clorpirifós (organofosforados), cujas estruturas químicas estão apresentadas na Figura 1, em tomate, utilizando a cromatografia gasosa de alta resolução acoplada a detector por captura de elétrons (CGAR-DCE). Dentre

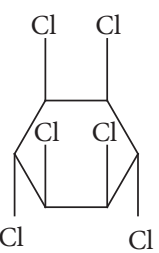

$\gamma-\mathrm{HCH}$<smiles>Cc1c(Cl)c(Cl)c(C#N)c(Cl)c1C#N</smiles>

$\mathrm{Cl}$

Clorotalonil

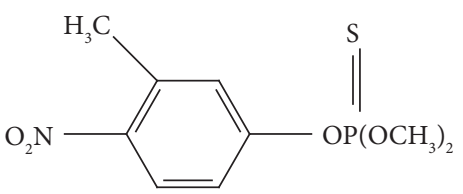

Fenitrotiona

Figura 1. Estruturas químicas dos cinco agrotóxicos estudados. os agrotóxicos estudados, apenas o clorotalonil, o clorpirifós e a procimidona são permitidos na cultura de tomates no Brasil em níveis de 1,0; 0,5; e 2,0 mg. $\mathrm{kg}^{-1}$, respectivamente (BRASIL, 2003). Vale salientar que outros resíduos podem ser encontrados em tomate em razão de uma aplicação indevida ou mesmo contaminação do meio ambiente. Sendo assim, a pesquisa de outros agrotóxicos não deve ser descartada.

\section{Material e métodos}

Para execução deste trabalho, vale salientar que as vidrarias utilizadas, pipetas volumétricas e balões volumétricos, apresentam certificado de calibração rastreáveis à Rede Brasileira de Calibração.

A balança analítica, com resolução de $10^{-5} \mathrm{~g}$, utilizada para pesagem dos padrões de agrotóxicos e da amostra se encontra devidamente calibrada, bem como o equipamento bem como o equipamento CG/DCE.

\subsection{Amostras}

Aproximadamente $2 \mathrm{~kg}$ de tomate da variedade Styllus (tomate longa vida híbrido Styllus), provenientes da região serrana do Estado do Rio de Janeiro, foram adquiridos de uma produção orgânica certificada com o selo de qualidade ABIO Associação de Agricultores Biológicos do Estado do Rio de Janeiro (ABIO, 2006).

Os tomates foram cortados em quatro partes e processados em blender (liquidificador com copo de aço inox) de acordo com indicação do Codex Alimentarius (CODEX, 2003), e a polpa foi mantida em recipientes de vidro até o momento da análise. Primeiramente a polpa de tomate foi testada e analisada com a finalidade de verificar a ausência dos agrotóxicos alvos do estudo.

\subsection{Padrões e reagentes}

Foram utilizados padrões de agrotóxicos com certificado de análise e grau de pureza superior a 95\% (Dr. Ehrenstorfer Augsburg, Alemanha). Solventes de alta pureza e grau resíduos de pesticidas (Merck e Tedia), acetona, diclorometano, éter de petróleo (faixa de ebulição $40-60^{\circ} \mathrm{C}$ ), iso-octano e acetato de etila foram utilizados. Sulfato de sódio anidro (granulado para análise de resíduos, Merck) e papel de filtro (Whatman $n^{\circ} 40$, diâmetro de $12,5 \mathrm{~cm}$ ) foram tratados de acordo com MorelliCardoso et al., 1999.

\section{Soluções estoque}

Soluções estoque na concentração nominal de $100 \mu \mathrm{g} \cdot \mathrm{mL}^{-1}$ foram preparadas individualmente por dissolução de cerca de 0,01 g do padrão de agrotóxico em $100 \mathrm{~mL}$ de solvente orgânico, iso-octano para $\gamma$ - $\mathrm{HCH}$, clorotalonil e procimidona, e em acetato de etila para a fenitrotiona, clorpirifós e clorpirifós metil.

\section{Soluções intermediárias}

Foram preparadas soluções intermediárias de trabalho contendo a mistura com os cinco agrotóxicos, estudados nas faixas de concentrações apresentadas na Tabela 1, em iso-octano a partir 
de diluições das soluções estoque preparadas previamente. Essas soluções foram empregadas para preparo das curvas analíticas (curva de calibração) em solvente iso-octano e no extrato orgânico da polpa de tomate bem como para fortificação da polpa de tomate no estudo da taxa de recuperação.

Para preparação da curva na matriz, $1 \mathrm{~mL}$ do extrato orgânico do branco da polpa de tomate foi evaporado sob atmosfera de $\mathrm{N}_{2}$ em bloco de aquecimento acoplado à unidade de evaporação e, após a secura, dissolvido em $1 \mathrm{~mL}$ da mistura da solução intermediária de agrotóxico com concentração correspondente ao ponto da curva analítica. Esse procedimento foi realizado para cada ponto da curva em questão.

\section{Solução intermediária do controlador individual do processo}

O clorpirifós metil atua como controlador individual do processo - CIP, que é uma substância com características físicas e químicas similares às dos agrotóxicos que estão sendo analisados, com o objetivo de garantir a integridade de uma amostra dentro do processo analítico (USDA, 2008). Quando a recuperação encontrada para esta substância estiver entre $70 \mathrm{e}$ $120 \%$, a integridade da amostra está garantida. Caso contrário deve-se verificar todo o procedimento.

A solução contendo o controlador individual do processo foi preparada na concentração nominal de $2 \mu \mathrm{g} \cdot \mathrm{mL}^{-1} \mathrm{em}$ solvente acetato de etila, a partir da referida solução estoque, e na concentração nominal de $0,02 \mu \mathrm{g} \cdot \mathrm{mL}^{-1}$ em solvente isooctano, a partir desta solução intermediária. A concentração de $0,02 \mu \mathrm{g} \cdot \mathrm{mL}^{-1}$ corresponde à solução intermediária a ser injetada no cromatógrafo a gás com detecção por captura de elétrons (CG/DCE).

\subsection{Condições cromatográficas}

Cromatógrafo a gás HP 6890 (Agilent), equipado com detector por captura de elétrons $\left(\mathrm{Ni}^{63}\right)$, sistema de injeção automático e estação de trabalho - ChemStation. Temperatura do injetor e detector de 210 e $300^{\circ} \mathrm{C}$, respectivamente. Coluna $5 \%$ fenil metil polisiloxano - HP 5MS de $30 \mathrm{~m}$ de comprimento; $0,25 \mathrm{~mm}$ de diâmetro interno e $0,25 \mu \mathrm{m}$ de espessura de filme. Programação de temperatura do forno de $80{ }^{\circ} \mathrm{C}(0 \text { minuto })^{\circledR 30} 0^{\circ} \mathrm{C} /$ minuto $180^{\circ} \mathrm{C}$ (8 minutos $)^{\oplus 2}{ }^{\circ} \mathrm{C} /$ minuto $200{ }^{\circ} \mathrm{C}(5 \text { minutos })^{\oplus 6}{ }^{\circ} \mathrm{C} /$ minuto $280{ }^{\circ} \mathrm{C}$ (5 minutos). Fluxo de gás carreador (Hélio) $=1,2 \mathrm{~mL} /$ minuto, fluxo da purga do septo $=2,8 \mathrm{~mL} /$ minuto, fluxo total $=64,1 \mathrm{~mL} /$ minuto, fluxo do gás make-up (Nitrogênio) $=60 \mathrm{~mL} /$ minuto, modo de injeção splitless $=0,75$ minutos, volume injetado $=1,0 \mu \mathrm{L}$. Tempo total de corrida de 45 minutos e integração pelo parâmetro área dos picos.

Tabela 1. Concentrações das soluções preparadas para o estudo de validação do método de ensaio.

\begin{tabular}{cc}
\hline Agrotóxico & Misturas soluções intermediárias $\left(\mu \mathrm{g} \cdot \mathrm{mL}^{-1}\right)$ \\
\hline$\gamma$-HCH & 0,003 a 3,018 \\
Clorotalonil & 0,003 a 15,585 \\
Fenitrotiona & 0,003 a 4,436 \\
Clorpirifós & 0,003 a 7,147 \\
Procimidona & 0,004 a 37,250 \\
\hline
\end{tabular}

\subsection{Procedimento analítico multirresíduos}

O método multirresíduos validado foi baseado nos procedimentos do Laboratório da Califórnia - Department of Food and Agriculture - Sacramento/CA (LEE et al., 1991) e de Laboratórios do Working Group on the Improvement and Development of Residue Methods - Netherlands (GENERAL INSPECTORATE FOR HEALTH PROTECTION, 1996), o qual se baseia na extração de diferentes resíduos de agrotóxicos nos hortifrutigranjeiros por solventes orgânicos, seguida de uma separação de fases líquida-líquida, evaporação e determinação dos agrotóxicos por cromatografia gasosa acoplada a detector por captura de elétrons.

\section{Extração dos agrotóxicos}

Foram pesadas $15 \mathrm{~g}$ da amostra processada em frasco para uso em centrífuga de $250 \mathrm{~mL}$, e $1 \mathrm{~mL}$ do controlador individual do processo (clorpirifós metil) na concentração nominal de $2 \mu \mathrm{g} \cdot \mathrm{mL}^{-1}$ foi adicionado à amostra antes da extração.

A extração foi realizada com a adição de $30 \mathrm{~mL}$ de acetona à amostra, que foi homogeneizada em agitador de alta dispersão por 30 segundos. Em seguida, adicionaram-se $60 \mathrm{~mL}$ de uma mistura diclorometano:éter de petróleo (1:1, v:v), e novamente feita a homogeneização em agitador de alta dispersão por 30 segundos.

Após a homogeneização, o frasco foi lacrado e levado para centrifugação por 7 minutos a $5{ }^{\circ} \mathrm{C}$ e $3000 \mathrm{rpm}$.

Em seguida, o extrato foi filtrado sob sulfato de sódio e papel de filtro, previamente lavado, sendo coletado em proveta graduada. Desse volume recolhido, retirou-se uma alíquota de $1 \mathrm{~mL}$ que foi transferida para "vial" de vidro e levada à evaporação, em bloco de aquecimento acoplado à unidade de evaporação, até à secura sob leve atmosfera de nitrogênio. $\mathrm{O}$ extrato seco foi dissolvido em $1 \mathrm{~mL}$ de iso-octano e $1 \mu \mathrm{L}$ analisado por CG/DCE.

A determinação do teor de agrotóxico na amostra é calculada por interpolação da área dos picos cromatográficos obtidos na curva analítica seguida da fórmula representada na Equação 1:

$$
\begin{aligned}
& \text { Resultado }\left(\mu \mathrm{g} \cdot \mathrm{g}^{-1} \text { ou mg.kg }{ }^{-1}\right)= \\
& \text { conc. obtida pela curva }\left(\mu \mathrm{g} \cdot \mathrm{mL}^{-1} \text { ou mg. } \mathrm{L}^{-1}\right) \times \\
& \times \frac{\text { vol. de extração }(90 \mathrm{~mL})+\text { vol. do CIP }(1 \mathrm{~mL})}{\text { massa pesada da matriz }(\mathrm{g})}
\end{aligned}
$$

em que: CIP representa o controlador individual do processo.

\subsection{Validação da metodologia e avaliação dos critérios de desempenho}

A validação do método foi conduzida segundo várias indicações citadas em literaturas científicas (CODEX, 2003; DG-SANCO, 2007; EURACHEM, 1998; BRASIL, 2007; MILLER, J. C.; MILLER, J. N., 1984; THOMPSON; ELLISON; WOOD, 2002). Isto foi feito através do cumprimento de alguns critérios de desempenho que são expressos em termos de parâmetros 
estatísticos de seletividade, linearidade, repetitividade, recuperação e limites de detecção e de quantificação.

\section{Seletividade}

A seletividade foi verificada através da análise de uma amostra de tomate (branco do tomate) que não contivesse os agrotóxicos em questão e que não apresentasse interferentes que coincidissem com os tempos de retenção das substâncias estudadas. Para comprovar a seletividade do método, foram injetadas misturas de padrões para identificação dos tempos de retenção de cada substância (DG-SANCO, 2007; RIBANI et al., 2004; THOMPSON; ELLISON; WOOD, 2002).

\section{Estudo da linearidade e faixa de trabalho}

O estudo da linearidade da faixa de trabalho foi realizado com a construção de curvas analíticas, preparadas em solvente (iso-octano) e na matriz (extrato orgânico do branco da polpa de tomate) nas concentrações nominais de 0,$003 ; 0,005 ; 0,01$; 0,$05 ; 0,1 ; 0,2$; e $0,4 \mu \mathrm{g} \cdot \mathrm{mL}^{-1}$, em duas replicatas genuínas de cada nível. As soluções da curva foram injetadas 2 vezes, para cada "vial", no cromatógrafo. A faixa de trabalho estabelecida está de acordo com a sensibilidade do método e o LMR dos agrotóxicos avaliados correspondendo às concentrações entre 0,02 e 2,5 mg. $\mathrm{kg}^{-1}$ de cada agrotóxico na polpa de tomate.

A linearidade da faixa de trabalho para o método de ensaio foi verificada através da leitura da curva analítica, utilizando o método dos mínimos quadrados ordinários (MMQO) de acordo com a relação linear representada pela Equação 2 tipo:

$y=b x+a$

A correlação representada pelo modelo matemático entre os valores numéricos de $\mathrm{x}$ e de y é representada pelo coeficiente de Pearson - 'r'. O quadrado deste coeficiente é chamado de coeficiente de determinação ou simplesmente $\mathrm{R}^{2}$. Vale ressaltar que o coeficiente de correlação - 'r' e o coeficiente de determinação - ' $\mathrm{R}^{2}$ ' são equivocadamente interpretados para avaliação da linearidade, não sendo adequados para este fim e não devem ser empregados isoladamente (THOMPSON; ELLISON; WOOD, 2002). Tais coeficientes indicam apenas o grau de ajuste dos dados à curva. Neste trabalho, os valores aceitos para este parâmetro foram de $\mathrm{R}^{2} \geq 0,95$ e de $\mathrm{r} \geq 0,98$.
Os resíduos decorrentes dos ajustes da curva analítica obtida através do MMQO são examinados de acordo com as premissas apresentadas na Tabela 2, a fim de verificar se há valores discrepantes, se apresentam comportamento homocedástico, se a equação de regressão é estatisticamente significativa e se há desvio de linearidade (EURACHEM , 1998; GREEN, 1996; BRASIL, 2007; PIMENTEL; NETO, 1996; RIBANI et al., 2004; THOMPSON; ELLISON; WOOD, 2002).

\section{Efeito da resposta cromatográfica} acentuada e induzida pela matriz

O efeito da resposta cromatográfica acentuada e induzida pela matriz ou "efeito matriz" observado pode causar um aumento ou diminuição da resposta do detector de um analito presente no extrato da amostra, quando comparado ao mesmo analito presente em solvente orgânico, gerando, desse modo, uma resposta errada (BRUCE; MINKKINEN; RIEKKOLA, 1998; EURACHEM, 1998; HAJŠLOVA et al., 1998; THOMPSON; ELLISON; WOOD, 2002; ZROSTLÍKOVÁS et al., 2001).

Para avaliação deste efeito, são estudadas curvas analíticas preparadas em solvente e na matriz polpa de tomate. $\mathrm{O}$ procedimento bem como as estatísticas e critérios de aceitação para avaliação deste efeito está apresentado detalhadamente por Cardoso, Nóbrega e Abrantes (2008).

\section{Limites de detecção e de quantificação do método}

Os limites de detecção do método (LDM) bem como os limites de quantificação do método (LQM) foram estabelecidos com base no método da relação sinal/ruído (S/R) em que se aceita a estimativa de 3:1, ou seja, o sinal produzido através da resposta da injeção de uma concentração conhecida do analito de interesse é 3 vezes maior do que o sinal produzido do ruído da linha de base do cromatograma (RIBANI et al., 2004; THOMPSON; ELLISON; WOOD, 2002). Esta relação (S/R) foi calculada através da ChemStation do cromatógrafo HP-6890.

Para estabelecer esta relação para os valores dos LDM e LQM, uma amostra da polpa de tomate foi pesada e fortificada com $1 \mathrm{~mL}$ de uma mistura contendo os agrotóxicos estudados, na concentração nominal de $1,0 \mu \mathrm{g} \cdot \mathrm{mL}^{-1}$, e extraída pelo método multirresíduos apresentado previamente. Vale salientar que, para a avaliação destes parâmetros, não é necessária a adição do

Tabela 2. Premissas para verificação da linearidade da faixa da curva analítica (EURACHEM , 1998; GREEN, 1996; BRASIL, 2007; PIMENTEL; BARROS-NETO, 1996; RIBANI et al., 2004; THOMPSON; ELLISON; WOOD, 2002).

\begin{tabular}{|c|c|c|}
\hline Avaliações & Estatística & Critérios de aceitação \\
\hline $\begin{array}{l}\text { 1. existência de valores discrepantes, } \\
\text { para cada nível de concentração }\end{array}$ & teste de Grubbs & $\mathrm{G}_{\text {calculado }}<\mathrm{G}_{\text {tabelado }}$ \\
\hline $\begin{array}{l}\text { 2. homogeneidade na variância } \\
\text { dos resíduos da regressão }\end{array}$ & teste de Cochran & $\mathrm{C}_{\text {calculado }}<\mathrm{C}_{\text {tabelado }(\mathrm{k}=7 ; \mathrm{n}=2 ; \alpha=0,05)}$ \\
\hline 3. significância da regressão & $\begin{array}{l}\text { Análise da variância } \\
\text { (ANOVA) } \\
\text { Teste F }\end{array}$ & $\begin{array}{l}\mathrm{F}_{\text {calculado }} \geq \text { valor-p, existe relação linear entre as variáveis e a inclinação da reta } \\
\text { de regressão não é nula. Há indicação de que a regressão é significativa. } \\
\mathrm{F}_{\text {calc }} \leq \text { valor-p não há indicação de existência de relação linear entre as variá- } \\
\text { veis x e y e não tem sentido utilizar a regressão. }\end{array}$ \\
\hline $\begin{array}{l}\text { 4. desvio da linearidade } \\
\text { da faixa de trabalho }\end{array}$ & $\begin{array}{l}\text { Análise da variância } \\
\quad \text { (ANOVA) } \\
\text { teste da falta de ajuste }\end{array}$ & $\begin{array}{l}\alpha=0,05 \leq \text { valor-p, se aceita a linearidade, o modelo é satisfatório. } \\
\alpha=0,05 \geq \text { valor-p, deve-se estabelecer outro intervalo para faixa de trabalho. }\end{array}$ \\
\hline
\end{tabular}


controlador individual do processo. O extrato orgânico obtido foi injetado seis vezes $(n=6)$ e os resultados, correspondendo às concentrações injetadas de $0,01006 \mu \mathrm{g} \cdot \mathrm{mL}^{-1}$ para o $\gamma$-HCH; $0,01039 \mu \mathrm{g} \cdot \mathrm{mL}^{-1}$ para o clorotalonil; $0,01109 \mu \mathrm{g} \cdot \mathrm{mL}^{-1}$ para a fenitrotiona; $0,01021 \mu \mathrm{g} . \mathrm{mL}^{-1}$ para o clorpirifós; e $0,01242 \mu \mathrm{g} \cdot \mathrm{mL}^{-1}$ para a procimidona, foram utilizados para estabelecer a relação (S/R). Em seguida, foram determinadas as médias e os desvios padrão das respostas, expressas em áreas, obtidas das replicatas de injeções realizadas. Os valores dos limites são calculados através das Equações 3 e 4:

$\operatorname{LDM}\left(\mu \mathrm{g} \cdot \mathrm{mL}^{-1}\right)=\frac{\text { concentração } \mathrm{S} / \mathrm{R} 3: 1 \times 3 \times \mathrm{S}}{\overline{\mathrm{X}}}$
$\operatorname{LQM}\left(\mu \mathrm{g} \cdot \mathrm{mL}^{-1}\right)=\frac{\text { concentração } \mathrm{S} / \mathrm{R} 3: 1 \times 10 \times \mathrm{S}}{\overline{\mathrm{X}}}$

onde, concentração S/R 3:1 é a concentração injetada que produziu uma resposta na razão sinal/ruído $(\mathrm{S} / \mathrm{R})$ de aproximadamente 3 sobre o ruído da linha base; $\overline{\mathrm{X}}$ corresponde à média das respostas medidas (áreas); e $S$ corresponde ao desvio padrão das respostas medidas (áreas). Para o cálculo da concentração correspondente em $\mu \mathrm{g} \cdot \mathrm{g}^{-1}$ ou mg. $\mathrm{kg}^{-1}$, utiliza-se a Equação 1.

Para efeito de confirmação dos valores de LQM calculados, foram preparadas seis replicatas da polpa de tomate nas respectivas concentrações e extraídas de acordo com o método de ensaio.

\section{Recuperação e repetitividade}

A realização dos experimentos de recuperação proporcionou estimar a recuperação do método através da comparação da concentração real de cada agrotóxico adicionado à amostra antes do procedimento de extração, com aquela encontrada após esta etapa (DG-SANCO, 2007).

No estudo da recuperação dos agrotóxicos da amostra de tomate, $1 \mathrm{~mL}$ de uma solução contendo os cinco agrotóxicos foram adicionados a $15 \mathrm{~g}$ da polpa de tomate branco. As amostras fortificadas, preparadas em seis replicatas de 3 diferentes níveis de concentrações, nível baixo equivalente ao LQM calculado, intermediário e alto $(\mathrm{n}=6$, total $\mathrm{n}=18)$, foram misturadas com bastão de vidro visando garantir sua homogeneidade. Depois disso, as amostras foram mantidas por 15 minutos em temperatura ambiente antes do procedimento de extração multirresíduos, para evaporação do solvente. As concentrações dos agrotóxicos correspondentes na amostra foram: $\gamma-\mathrm{HCH}$ de 0,02; 0,10 e 0,20 mg. $\mathrm{kg}^{-1}$; clorotalonil de 0,02; 0,10 e 1,00 mg.kg-1; fenitrotiona de 0,$03 ; 0,10$ e $0,30 \mathrm{mg} \cdot \mathrm{kg}^{-1}$; clorpirifós de 0,02 ; 0,10 e $0,5 \mathrm{mg} \cdot \mathrm{kg}^{-1}$ e procimidona de 0,$02 ; 0,10$ e $2,5 \mathrm{mg} \cdot \mathrm{kg}^{-1}$. Os valores diferenciados no terceiro nível (nível alto) se devem ao fato de se avaliarem níveis próximos ao LMR estabelecido do agrotóxico no tomate. Os valores do nível um (nível baixo) foram utilizados para confirmação dos LQM calculados através da razão sinal/ruído (S/R). A fórmula utilizada para o cálculo da taxa de recuperação está demonstrada na Equação 5:

Taxa de recuperação $(\%)=\frac{\overline{\text { Xexperimental }}}{\overline{\text { Xteórica }}} \times 100$

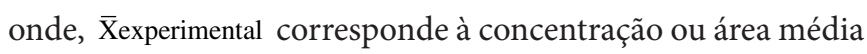
$(\mathrm{n}=6)$ obtida experimentalmente e X̄teórica corresponde à concentração ou área média teórica adicionada à amostra.

A precisão é medida sob condições de repetitividade ou sob condições de reprodutibilidade e expressa na forma de coeficiente de variação - (CV (\%)) e/ou estimativa do desvio padrão relativo - DPR (\%) (THOMPSON; ELLISON; WOOD, 2002; DG-SANCO, 2007). A precisão foi avaliada através da repetitividade dos experimentos de recuperação, de acordo com a Equação 6.

$\operatorname{DPR}(\%)$ ou $\operatorname{CV}(\%)=\frac{S}{\bar{X}} 100$

onde, $S$ corresponde ao desvio padrão das leituras no nível de concentração estudado e $\overline{\mathrm{X}}$ à média dos resultados obtidos através das replicatas $(n=6)$.

Como critério de aceitação dos parâmetros exatidão e precisão, foram adotados como referência os valores estabelecidos pelo Codex (2003) e DG-Sanco (2007).

\section{Resultados e discussão}

A amostra de tomate orgânico testemunha apresentou-se satisfatória para avaliação do método realizado, já que não continha os cinco agrotóxicos estudados e nenhum interferente de coeluição que impedisse a identificação e quantificação deles.

\subsection{Validação da metodologia e avaliação dos critérios de desempenho}

\section{Seletividade}

Na Figura 2, são apresentados os cromatogramas do branco da amostra (amostra testemunho) e da mistura padrão. Os picos que apareceram no branco da amostra não coincidem com os dos cinco agrotóxicos em questão, não interferindo na análise com as condições cromatográficas determinadas, consequentemente demonstrando que a polpa de tomate adquirida se encontra em condições favoráveis à fortificação das substâncias em estudo.

\section{Estudo da linearidade e faixa de trabalho}

A avaliação da curva analítica pelo MMQO foi realizada através de uma planilha eletrônica para cada substância estudada. A Figura 3 apresenta o modelo da planilha com os dados obtidos para o $\gamma$ - $\mathrm{HCH}$ na polpa de tomate (curva preparada na matriz).

O uso de planilhas para cálculos em uma validação tornouse uma necessidade na comunidade científica tendo em vista a complexidade dos cálculos empregados e a redução do tempo de processamento de dados. Essas planilhas possibilitam a execução de cálculos matemáticos e estatísticos bem como a apresentação dos resultados em tabelas e gráficos. Contudo, nem sempre esses programas computacionais existentes dispõem de todas as facilidades requeridas à avaliação de um ensaio específico. Cabe ao laboratório analítico definir qual ferramenta computacional 

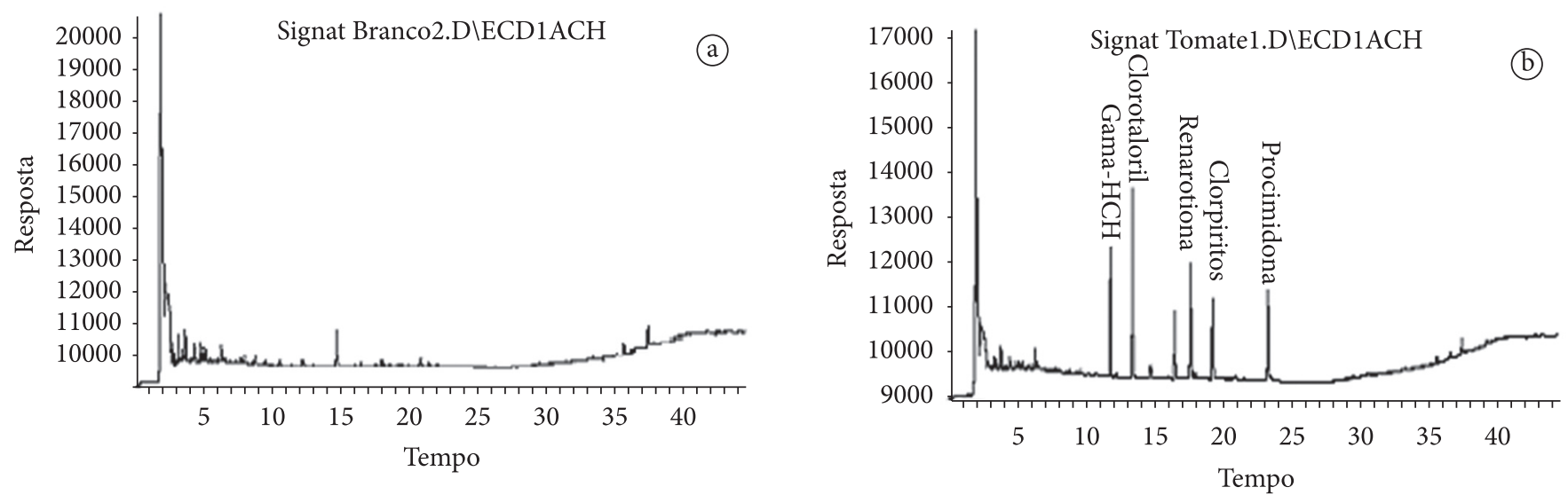

Figura 2. A - Cromatograma do branco da polpa de tomate analisada por CG/DCE, de acordo com as condições cromatográficas estabelecidas previamente. B - Cromatograma da polpa de tomate fortificada com uma mistura de: $\gamma$-HCH, clorotalonil, fenitrotiona, clorpirifós e procimidona correspondendo às concentrações de $0,01006 \mathrm{mg} \cdot \mathrm{kg}^{-1} ; 0,01039 \mathrm{mg} \cdot \mathrm{kg}^{-1} ; 0,01109 \mathrm{mg} \cdot \mathrm{kg}^{-1} ; 0,01021 \mathrm{mg} \cdot \mathrm{kg}^{-1} ;$ e $0,01242 \mathrm{mg} \cdot \mathrm{kg}^{-1}$, respectivamente, analisada por CG/DCE.

Laboratório de resíduos de agrotóxicos

Avaliação da curva analítica do agrotóxico gama-HCH na matriz Tomate

\begin{tabular}{|c|c|c|c|c|c|c|c|c|c|c|c|c|c|}
\hline \multicolumn{3}{|l|}{ Data: } & \multicolumn{4}{|c|}{ Equipamento: } & \multicolumn{5}{|l|}{ Sequência: } & & \\
\hline \multicolumn{3}{|c|}{ Curva de calibração } & \multicolumn{4}{|c|}{ Cálculos } & \multicolumn{5}{|c|}{ Avaliação dos resíduos - Homocedasticidade } & & \\
\hline \multicolumn{2}{|c|}{ Concentração } & \multirow{3}{*}{$\begin{array}{l}\text { Resposta } \\
\text { área } \\
56454.5\end{array}$} & \multirow{3}{*}{$\begin{array}{l}\text { Média } \\
\quad 55407\end{array}$} & \multirow{3}{*}{$\begin{array}{l}\text { Variância } \\
\text { 2194512,5 }\end{array}$} & \multirow{3}{*}{$\begin{array}{l}\text { Desv. Pad. } \\
1481,3887\end{array}$} & \multirow{3}{*}{$\begin{array}{c}\mathrm{CV} \\
\% \\
2,674\end{array}$} & \multirow{3}{*}{\begin{tabular}{|c|} 
Somatório \\
Variância \\
$1,13767 \mathrm{E}+11$
\end{tabular}} & \multirow{3}{*}{$\begin{array}{c}\text { Maior } \\
\text { Variãncia } \\
50811619328\end{array}$} & \multicolumn{2}{|c|}{ C de Cohran } & \multirow{3}{*}{\begin{tabular}{|c|} 
Resultado \\
homo/hetero \\
Homocedástico
\end{tabular}} & & \\
\hline Pontos & $\mu \mathrm{g} \cdot \mathrm{mL}^{-1}$ & & & & & & & & \multirow{3}{*}{\begin{tabular}{|c} 
C calculado \\
0,4466
\end{tabular}} & & & & \\
\hline 1 & 0,0030 & & & & & & & & & & & & \\
\hline \multirow[b]{2}{*}{2} & $\begin{array}{l}0,0030 \\
0,0050\end{array}$ & $\begin{array}{r}54359,5 \\
99814 \\
\end{array}$ & \multirow{2}{*}{100542,25} & \multirow{2}{*}{1060696,125} & \multirow{2}{*}{1029,9010} & \multirow{2}{*}{1,024} & \multicolumn{3}{|c|}{ ANOVA - da regressão e Teste de desvio da Linearidade } & \begin{tabular}{|c|} 
C teórico \\
0,7270
\end{tabular} & & & \\
\hline & 0,0050 & 101270,5 & & & & & Fonte & G.L. & SQ & MQ & $\mathrm{F}$ & valor-p & Sign. \\
\hline \multirow{2}{*}{3} & 0,0101 & 166554,5 & 1675105 & 1827872 & 13519802 & 0807 & Regressão & 1 & $2,24 \mathrm{E}+14$ & $2,24 \mathrm{E}+14$ & 9405,2614 & 9,662E-19 & $\mathrm{p}<0,05$ \\
\hline & 0,0101 & 168466,5 & 167510,5 & $182 / 8 / 2$ & 1351,9882 & 0,807 & ResÍduos & 12 & $2,85 \mathrm{E}+11$ & $2,38 \mathrm{E}+10$ & $\#$ & & \\
\hline 4 & 0,0503 & 1068151 & 10024635 & 1182195312 & & & Falta de ajuste & 5 & $1,71 \mathrm{E}+11$ & $3,43 \mathrm{E}+10$ & 2,1104 & 0,1788 & $\mathrm{p}>0,05$ \\
\hline 4 & 0,0503 & 1116776,0 & 1092463,5 & 1182195313 & 34383,0672 & 3,147 & Erro puro & 7 & $1,14 \mathrm{E}+11$ & $1,63 \mathrm{E}+10$ & \# & & \\
\hline 5 & 0,1006 & 2682161,5 & 2760624.5 & 12312884738 & 1109634387 & 4020 & Total & 13 & $2,24 \mathrm{E}+14$ & \# & \# & & \\
\hline & 0,1006 & 2839087,5 & & $12312884 / 38$ & 110963,438 & 4,020 & Xmed $=$ & 0,1104 & & & & & \\
\hline 6 & 0,2012 & 5546854,0 & 5706246 & 50811619328 & 225414.3281 & 3.950 & Ymed $=$ & 3084213,36 & & & & & \\
\hline & 0,2012 & 5865638,0 & 5706246 & 50811619328 & 225414,3281 & 3,950 & $\mathrm{Se}^{2}=$ & 23772113753,30 & & & Significativa & & \\
\hline 7 & 0,4024 & 11549450,0 & 11706699,75 & 49454967750 & 222384,7291 & 1.900 & $\mathrm{Se}=$ & 154182,08 & & & & & \\
\hline & 0,4024 & 11863949,5 & & & & 1,900 & $\mathrm{n}=$ & 14 & Resultado & Regressão & & & \\
\hline Somatório & 1,5452 & \begin{tabular}{|l|l|}
43178987 \\
\end{tabular} & & & & & coef.linear $=$ & $-153376,5222$ & & & & & \\
\hline & & & & & & & coef.ang $=$ & 29333586,7921 & & & Não há desvio & & \\
\hline & & & & & & & alfa $=$ & 0,05 & & & & & \\
\hline & & & & & & & $\mathrm{u}=$ & 7 & & & & & \\
\hline & & & & & & & $\mathrm{r}=$ & 0,9994 & $\mathrm{n}=$ número & e observações & & & \\
\hline & & Curv & a analítica & ama-HCl & $\mathrm{em}$ & & $\mathrm{R} 2=$ & 0,9987 & $\mathrm{u}=$ número & e níveis & & & \\
\hline 140000 & & matri & $\mathrm{z}$ tomate - & em dupl & & & $\mathrm{P}=$ & 0,05 & $2=$ número & e parâmetros & estimados & & \\
\hline 120000 & & $=29333$ & $7921 \times$ & 3376,5 & & & & & & & & & \\
\hline 100000 & & & & & & & & & & & & & \\
\hline ङ 80000 & & & & & & & & & Grá & co de resíd & luos & & \\
\hline 40000 & & & & & & & $\begin{array}{l}300000 \\
200000 \\
100000\end{array}$ & $\bullet$ & & & & $\nabla+7$ & Y \\
\hline 20000 & & & & & & $\neg$ & $\begin{array}{r}100000 \\
-1000000\end{array}$ & & & $\bullet$ & $\bullet$ & $\checkmark$ & \\
\hline & 0 & 0,1 & 0,2 & 0,3 & 0,4 & 0,5 & $-400000 亡$ & & & & & & \\
\hline & & Conce & ntração col & hhecida (ug. & $\left.\mathrm{mL}^{-1}\right)$ & & 1 & 23 & 5 & $\begin{array}{ll}7 & 8\end{array}$ & $\begin{array}{lll}9 & 10 & 11\end{array}$ & 1213 & 14 \\
\hline
\end{tabular}

Figura 3. Planilha eletrônica para avaliação da linearidade da curva analítica. Modelo referente à curva analítica na matriz tomate para o $\gamma$-HCH, com sete níveis de concentrações em duplicata. 
utilizará para avaliar seus dados, entretanto a possibilidade do desenvolvimento de uma planilha que satisfaça às necessidades do laboratório não deve ser descartada.

A planilha em questão foi desenvolvida no programa Microsoft Excel ${ }^{\Phi}$ de modo a fornecer em uma única página a análise do gráfico $x-y$ (curva analítica construída com 7 níveis), o gráfico dos resíduos da regressão (observação de valores discrepantes), a variância dos resíduos (avaliação da homocedasticidade pelo teste de Cochran), a significância da regressão (aplicação da análise de variância - teste F) e se há desvio na linearidade da faixa de trabalho (Teste da falta de ajuste).

Os principais e únicos dados lançados na planilha correspondem aos valores das variáveis $\mathrm{x}$ e $\mathrm{y}$, ou seja, as concentrações da curva analítica e as respostas médias (áreas),
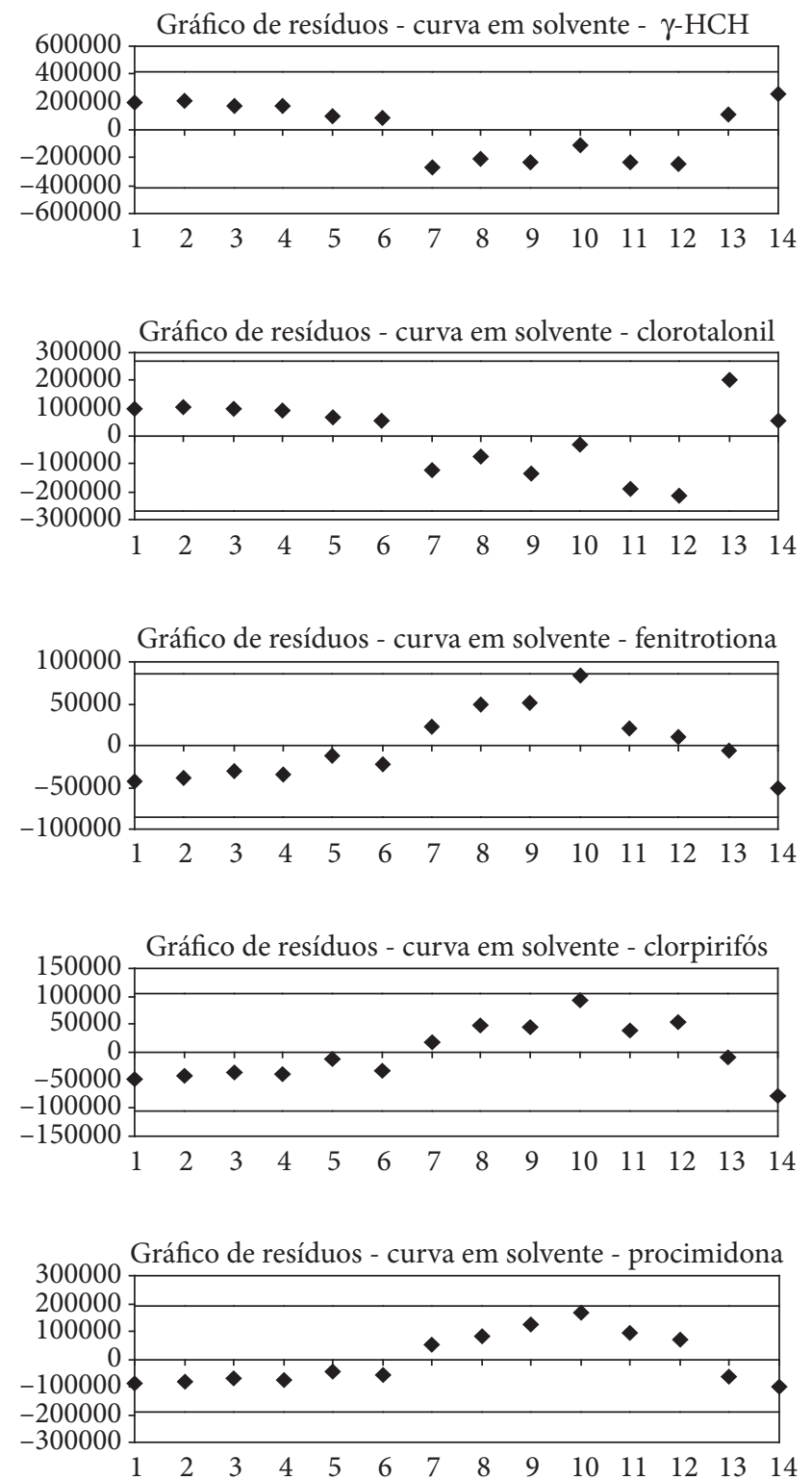

respectivamente, efetuando a partir destas informações digitadas os cálculos necessários à realização dos testes estatísticos citados na Tabela 2.

Vale salientar que o detalhamento da planilha eletrônica apresentada não é o foco deste trabalho, mas serve para ilustrar seu benefício para o objetivo proposto.

A planilha eletrônica utilizada para avaliação das premissas relacionadas à verificação da linearidade da faixa de trabalho mostrou-se bastante adequada ao roteiro de validação adotado no laboratório.

A Figura 4 apresenta os gráficos de resíduos da regressão obtidos para as substâncias estudadas. Não foram observados valores discrepantes em nenhuma situação apresentada.
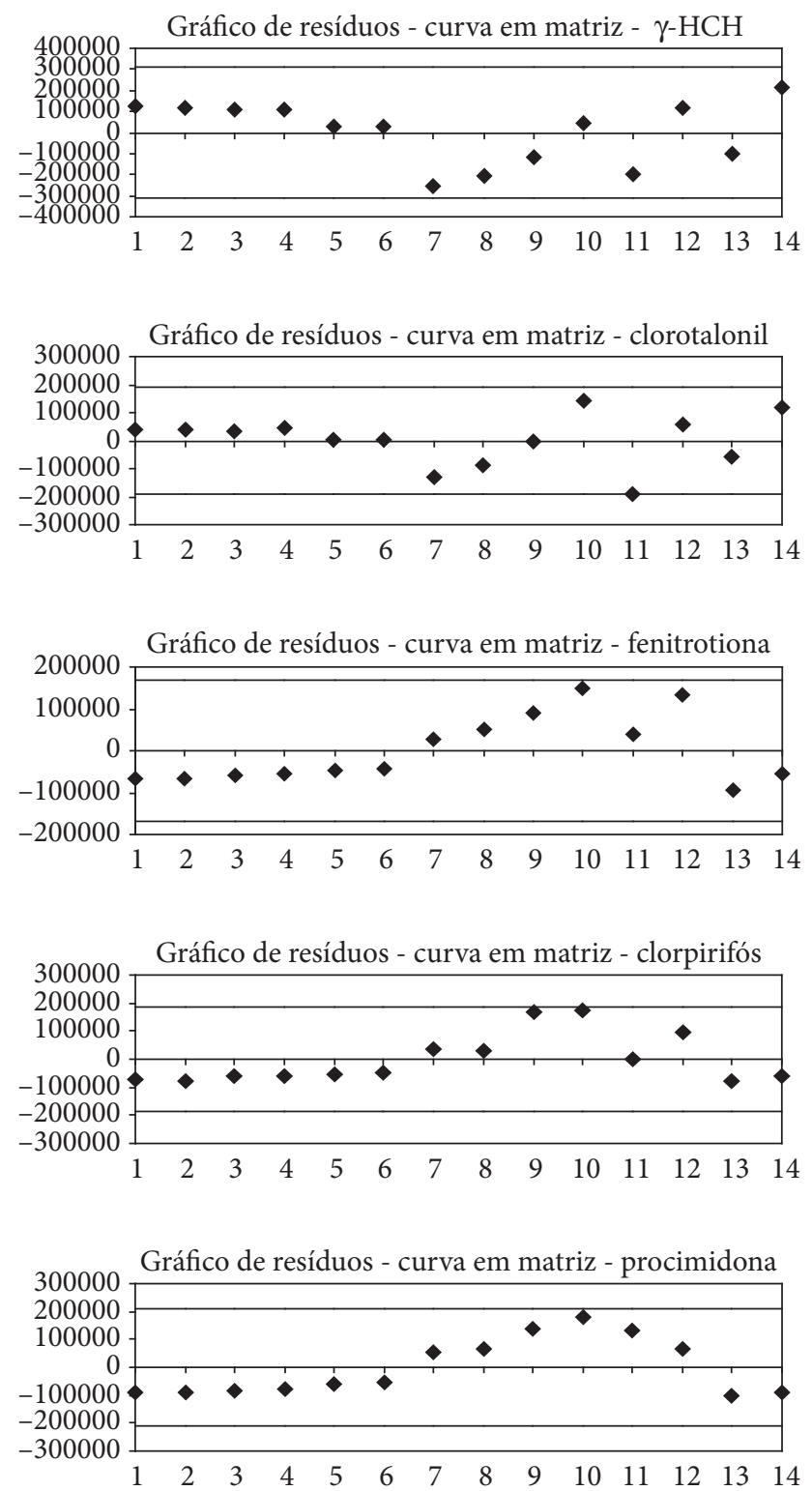

Figura 4. Representação gráfica dos resíduos da regressão das curvas analíticas construídas em solvente e na matriz para os cinco agrotóxicos estudados. 
O teste estatístico empregado para avaliar a variância dos resíduos provenientes dos ajustes das curvas analíticas em solvente e em matriz para os agrotóxicos, de acordo com a Tabela 2, demonstra que apresentam comportamento homocedásticos $\left(\mathrm{C}_{\text {calculado }}<\mathrm{C}_{\text {tabelado }}\right)$ conforme apresentado na Tabela 3.

Na Tabela 4, são apresentadas as equações da reta de regressão empregadas para verificação da significância da regressão e desvio da linearidade. É possível observar que a regressão foi significante não demonstrando desvio da linearidade na faixa de trabalho avaliada para os cinco agrotóxicos alvos do estudo. Entretanto, nas análises de rotina, adotou-se a utilização de curvas analíticas com apenas três a cinco níveis de concentrações diferentes devido à complexidade e ao custo da análise de resíduos de agrotóxicos em alimentos.

\section{Efeito da resposta cromatográfica}

acentuada e induzida pela matriz

De acordo com os resultados apresentados por Cardoso, Nóbrega e Abrantes (2008), o efeito matriz demonstrou ser

Tabela 3. Resultados da avaliação através do teste de Cochran, da homogeneidade da variância dos resíduos da regressão da curva analítica, em solvente e no extrato orgânico da polpa de tomate.

\begin{tabular}{lcccc}
\hline Agrotóxico & $\begin{array}{c}\mathrm{C}_{\text {calculado }} \\
\text { curva em } \\
\text { solvente }\end{array}$ & $\begin{array}{c}\mathrm{C}_{\text {calculado }} \\
\text { curva em } \\
\text { matriz }\end{array}$ & $\begin{array}{c}\mathrm{C}_{\text {tabelado }} \\
(\mathrm{k}=7, \mathrm{n}=2)\end{array}$ & Resultado \\
\hline $\boldsymbol{\gamma}$-HCH & 0,5530 & 0,4466 & 0,727 & homoscedástico \\
Clorotalonil & 0,6074 & 0,5380 & 0,727 & homoscedástico \\
Fenitrotiona & 0,5016 & 0,6171 & 0,727 & homoscedástico \\
Clorpirifós & 0,5397 & 0,6709 & 0,727 & homoscedástico \\
Procimidona & 0,3800 & 0,6411 & 0,727 & homoscedástico \\
\hline
\end{tabular}

Os resultados apresentados na tabela 3 referem-se à avaliação de duplicatas de

7 concentrações diferentes injetadas duas vezes cada uma. significante para os agrotóxicos da classe dos organofosforados, a fenitrotiona e o clorpirifós, indicando a utilização da curva analítica preparada com o extrato orgânico branco da polpa de tomate a fim de minimizar esse efeito. Embora para o $\gamma-\mathrm{HCH}$, o clorotalonil e a procimidona esse efeito não seja significativo, adotou-se como forma de trabalho o emprego da curva preparada na matriz para os cinco agrotóxicos alvos do estudo.

\section{Limites de detecção e de quantificação do método}

Os valores dos limites de detecção e dos limites de quantificação do método calculados através das Equações 3 e 4 são apresentados na Tabela 5. Apesar da relação sinal/ruído produzida pelo software ser superior a 3 , decidiu-se manter a concentração nominal de $0,01 \mu \mathrm{g} \cdot \mathrm{mL}^{-1}$ como base para cálculos do parâmetro limite de detecção do método. Esses parâmetros calculados através do método de ensaio são os que mais refletem os valores reais e foram adotados como referência neste trabalho. Os valores dos LQM foram confirmados através do teste realizado com seis replicatas da amostra fortificadas com as respectivas concentrações, além de serem inferiores ao LMR estabelecido para o clorotalonil, clorpirifós e procimidona em tomate, demonstrando a adequação do método à sua finalidade.

\section{Recuperação e repetitividade}

Segundo o documento do DG-Sanco 2007, a possibilidade de se calcular a taxa de recuperação com apenas um único nível de calibração pode ser empregada em situações em que a concentração investigada for muito baixa, fornecendo resultados mais exatos do que quando utilizados vários níveis de calibração. Desse modo, as taxas de recuperação encontradas para os níveis mais baixos seguiram essa indicação para os cinco agrotóxicos em questão. Para os níveis intermediário e alto, utilizou-se a padronização externa através da curva analítica construída com os sete níveis de concentrações diferentes.

Tabela 4. Equação da reta de regressão e coeficientes de determinação para as faixas de trabalho estudadas para cinco agrotóxicos por CG-DCE nas curvas em solvente e em extrato orgânico do tomate e estatísticas para avaliação da linearidade.

\begin{tabular}{|c|c|c|c|c|c|}
\hline \multirow[t]{2}{*}{ Agrotóxico e estatísticas } & \multirow{2}{*}{$\begin{array}{c}\text { Faixa linear } \\
\left(\mu \mathrm{g} \cdot \mathrm{mL}^{-1}\right)\end{array}$} & \multicolumn{2}{|l|}{ Curva em iso-octano } & \multicolumn{2}{|l|}{ Curva em extrato orgânico do tomate } \\
\hline & & Equação da regressão & $\mathrm{R}^{2}$ & Equação da regressão & $\mathrm{R}^{2}$ \\
\hline$\gamma-\mathrm{HCH}$ & & $y=30453162,67 x-251452,48$ & & $y=29333864,59 x-153440,71$ & \\
\hline ANOVA - teste $\mathrm{t}$ & $0,003-0,402$ & $\mathrm{~F}_{\text {calculado }}(5487,01)>$ valor-p $\left(2,43^{*} 10^{-17}\right)$ & 0,9978 & $\mathrm{~F}_{\text {calculado }}(9405,26)>$ valor-p $\left(9,66^{*} 10^{-19}\right)$ & 0,9987 \\
\hline ANOVA - falta de ajuste & & $\alpha=0,05<$ valor-p $(0,06)$ & & $\alpha=0,05<$ valor-p $(0,18)$ & \\
\hline clorotalonil & & $y=27585538,98 x-11,9526,43$ & & $y=27428493,32 x-49651,41$ & \\
\hline ANOVA - teste $\mathrm{t}$ & $0,003-0,416$ & $\mathrm{~F}_{\text {calculado }}(11868,40)>$ valor- $\mathrm{p}\left(2,40^{*} 10^{-19}\right)$ & 0,9990 & $\mathrm{~F}_{\text {calculado }}(22796,63)>$ valor- $\mathrm{p}\left(4,79^{*} 10^{-21}\right)$ & 0,9995 \\
\hline ANOVA - teste $\mathrm{t}$ & $0,003-0,444$ & $\mathrm{~F}_{\text {calculado }}(12160,35)>$ valor- $\mathrm{p}\left(2,07^{\star} 10^{-19}\right)$ & 0,9990 & $\mathrm{~F}_{\text {calculado }}(3496,66)>$ valor-p $\left(3,62^{\star} 10^{-16}\right)$ & 0,9966 \\
\hline ANOVA - falta de ajuste & & $\alpha=0,05<$ valor- $\mathrm{p}(0,15)$ & & $\alpha=0,05<$ valor- $\mathrm{p}(0,13)$ & \\
\hline clorpirifós & & $y=10340903,25 x+59079,28$ & & $y=10792912,27 x+147778,22$ & \\
\hline ANOVA - teste $\mathrm{t}$ & $0,003-0,408$ & $\mathrm{~F}_{\text {calculado }}(10678,03)>$ valor- $\mathrm{p}\left(4,52^{\star} 10^{-19}\right)$ & 0,9989 & $\mathrm{~F}_{\text {calculado }}(2900,62)>$ valor-p $\left(1,11^{\star} 10^{-15}\right)$ & 0,9959 \\
\hline ANOVA - falta de ajuste & & $\alpha=0,05<$ valor-p $(0,07)$ & & $\alpha=0,05<$ valor-p $(0,20)$ & \\
\hline
\end{tabular}


Tabela 5. Limites de detecção (LDM) e de quantificação (LQM) do método, calculados a partir da razão sinal/ruído, para o agrotóxico através do método de ensaio.

\begin{tabular}{|c|c|c|c|c|c|c|}
\hline \multirow[t]{2}{*}{ Agrotóxico } & \multirow[t]{2}{*}{$\begin{array}{c}\text { Massa de polpa } \\
\text { pesada (g) }\end{array}$} & \multirow[t]{2}{*}{$\begin{array}{l}\text { (a) (b) Relação sinal/ } \\
\text { ruído }\end{array}$} & \multirow[t]{2}{*}{$\begin{array}{l}{ }^{\text {(b) }} \text { Áreas } \\
\text { medidas }\end{array}$} & \multirow[t]{2}{*}{$\begin{array}{l}\text { (b)Desvio padrão } \\
\text { das áreas }\end{array}$} & \multicolumn{2}{|c|}{$\begin{array}{l}\text { (b) Limites do método }\left(\mu \mathrm{g} \cdot \mathrm{g}^{-1}\right) \\
\left(\mathrm{mg}^{-1} \mathrm{~kg}^{-1}\right)\end{array}$} \\
\hline & & & & & LDM & LQM \\
\hline$\gamma-\mathrm{HCH}$ & & 68,25 & 136175,83 & 3779,21 & 0,005 & 0,016 \\
\hline Clorotalonil & & 192,08 & 232753,00 & 7654,25 & 0,006 & 0,020 \\
\hline Fenitrotiona & 15,7593 & 81,25 & 153382,67 & 4379,16 & 0,005 & 0,018 \\
\hline Clorpirifós & & 107,48 & 110837,83 & 2663,65 & 0,004 & 0,014 \\
\hline Procimidona & & 194,35 & 156733,83 & 4378,45 & 0,006 & 0,020 \\
\hline
\end{tabular}

(a) valores obtidos através do software da ChemStation do cromatógrafo HP-6890. ${ }^{(\mathrm{b})}$ Dados foram obtidos através da média de seis determinações ( $\mathrm{n}=6$ ), realizadas em paralelo para: $\gamma$-HCH, clorotalonil, fenitrotiona, clorpirifós e procimidona obtidos a partir das análises cromatográficas por CG/DCE das concentrações injetadas de 0,01006, 0,01039, 0,01109, 0,01021 e $0,01242 \mathrm{mg} \cdot \mathrm{mL}^{-1}$, respectivamente.

Tabela 6. Parâmetros estatísticos de recuperação e de coeficiente de variação calculados para a matriz tomate, para $n=6$.

\begin{tabular}{|c|c|c|c|}
\hline Agrotóxico & $\begin{array}{l}\text { Concentração } \\
\text { de fortificação } \\
\left(\mathrm{mg} \cdot \mathrm{kg}^{-1}\right)\end{array}$ & $\begin{array}{c}\text { Recuperação } \\
(\%)\end{array}$ & $\begin{array}{l}\text { CV } \\
(\%)\end{array}$ \\
\hline \multirow[t]{3}{*}{$\gamma-\mathrm{HCH}$} & 0,020 & 77 & 9 \\
\hline & 0,100 & 86 & 9 \\
\hline & 0,200 & 75 & 15 \\
\hline \multirow[t]{3}{*}{ Clorotalonil } & 0,030 & 83 & 5 \\
\hline & 0,100 & 99 & 4 \\
\hline & 1,040 & 97 & 6 \\
\hline \multirow[t]{3}{*}{ Fenitrotiona } & 0,030 & 103 & 4 \\
\hline & 0,100 & 81 & 3 \\
\hline & 0,300 & 103 & 5 \\
\hline \multirow[t]{3}{*}{ Clorpirifós } & 0,020 & 72 & 6 \\
\hline & 0,100 & 72 & 4 \\
\hline & 0,500 & 92 & 6 \\
\hline \multirow[t]{3}{*}{ Procimidona } & 0,025 & 76 & 4 \\
\hline & 0,100 & 103 & 2 \\
\hline & 2,500 & 110 & 2 \\
\hline
\end{tabular}

Na Tabela 6, são apresentados as taxas de recuperação e os coeficientes de variação para os níveis de concentrações estudados, calculados através das Equações 5 e 6. Para todos os agrotóxicos estudados, as taxas de recuperações se apresentam na faixa aceitável de 70 a $120 \%$ com os respectivos CV (\%) inferiores a $20 \%$, estando de acordo com o recomendado pelo Codex Alimentarius (CODEX, 2003) e DG-Sanco, 2007.

Os critérios de aceitação indicados nestes documentos oficiais indicam que, para a faixa de concentração $>0,01 \mathrm{e} \leq 0,1 \mathrm{mg} \cdot \mathrm{kg}^{-1}$, o intervalo de recuperação aceitável em \% é entre 70-120 com CV (\%) de até $20 \%{ }^{9}$, > 0,1 e $\leq 1$ mg. $\mathrm{kg}^{-1}$ entre $70-110 \%$ e CV (\%) de até $15 \%$ e, para a faixa de concentração $>1 \mathrm{mg} \cdot \mathrm{kg}^{-1}$, o intervalo aceito se encontra entre $70-110 \%$ com CV $(\%) \leq 10 \%$ (CODEX, 2003); por outro lado, o DG-Sanco (2007) indica apenas a faixa de recuperação de 70-120\% com CV (\%) de até $20 \%$.

De modo a comprovar que, nos procedimentos adotados para a realização do método de ensaio estudado, houve a oportunidade de participação em dois ensaios de proficiência (EP) (THOMPSON; ELLISON; WOOD, 2006) provenientes do NMI-Austrália, no ano de 2006 e do FAPAS - Reino Unido, no ano de 2007. A matriz recebida foi a polpa de tomate que continha, entre outros, o agrotóxico procimidona. Os resultados encontrados nos dois EP, através do método de ensaio descrito neste trabalho, obtiveram como avaliação de desempenho o valor do índice $\mathrm{Z} \leq 2$, ou seja, satisfatório de acordo com a ISO Guia 43-1 (ABNT ISO/IEC, 1999) para este agrotóxico corroborando com a habilidade do método multirresíduos validado para esta substância.

\section{Conclusões}

O modelo proposto para verificação do desempenho do método de ensaio, demonstrando a qualidade e confiabilidade dos dados analíticos, seguiu parâmetros indicados na literatura para realização da validação e foi selecionado para atender às necessidades do trabalho realizado.

A utilização da planilha eletrônica para avaliação do parâmetro linearidade proporcionou rápida interpretação dos dados obtidos para a faixa analítica estudada demonstrando ser uma ferramenta aplicável às expectativas do laboratório.

Os resultados apresentados neste trabalho demonstraram a habilidade do método estudado e são satisfatórios para as necessidades da rotina do laboratório, já que é um procedimento multirresíduos, simples, de baixo custo por não precisar da etapa de limpeza (clean-up) e eficiente.

A oportunidade de participação em dois ensaios de proficiência em matriz tomate contendo o agrotóxico procimidona pode ainda comprovar a exatidão do método objeto de estudo para este analito.

\section{Referências bibliográficas}

ASSOCIAÇÃO BRASILEIRA DA INDÚSTRIA DA ALIMENTAÇÃO ABIA. Disponível em: <http://www.abia.org.br>. Acesso em: 06 jul. 2007.

ASSOCIAÇÃO BRASILEIRA DE NORMAS TÉCNICAS - ABNT. ABNT ISO/IEC Guia 43-1: 1999. Ensaio de Proficiência por comparações interlaboratoriais - Parte 1. Desenvolvimento e Operação de programas de Ensaios de Proficiência. Rio de janeiro: ABNT, 1999.

ASSOCIAÇÃO DE AGRICULTORES BIOLÓGICOS DO ESTADO DO RIO DE JANEIRO - ABIO. Disponível em: <http://www.abio. org.br>. Acesso em: 23 out. 2006. 
BRASIL. Ministério da Saúde. Agência Nacional de Vigilância Sanitária - ANVISA. Monografias de Produtos Agrotóxicos. 2003a. Disponível em: <http://www.anvisa.gov.br/toxicologia/ monografias/index.htm>. Acesso em: 06 jul. 2007.

BRASIL. Ministério do Desenvolvimento, Indústria e Comércio Exterior. Instituto Nacional de Metrologia, Normalização e Qualidade Industrial - INMETRO. Orientações sobre Validação de Métodos de Ensaios Químicos. DOQ-CGCRE-008. 2007. Disponível em: <http://www.inmetro.gov.br/Sidoq/Arquivos/ CGCRE/DOQ/DOQ-CGCRE-8_02.pdf>. Acesso em: 18 set. 2008.

BRASIL. Ministério do Planejamento, Orçamento e Gestão. Instituto Brasileiro de Geografia e Estatística - IBGE. Levantamento Sistemático da Produção Agrícola. Disponível em: <http://www. sidra.ibge.gov.br/bda/default.asp >. Acesso em: 08 out. 2008.

BRASIL. Resolução-RE n ${ }^{\circ} 165$, de 29 de agosto de 2003. Diário Oficial da República Federativa do Brasil, Brasília, DF, 02 set. 2003b. Seção 1, p. 48-50.

BRUCE, P.; MINKKINEN, P.; RIEKKOLA, M. L. Practical method validation: validation sufficient for an analysis method. Mikrochimica Acta, v. 128, n. 1-2, p. 93-106, 1998.

CARDOSO, M. H. W. M.; ABRANTES, S.; NÓBREGA, A. Estudo para preparação e certificação de um material de referência a ser usado no controle de agrotóxicos em hortifrutigranjeiros. In: JORNADA CIENTÍFICA DE PÓS-GRADUAÇÃO DA FUNDAÇÃO OSWALDO CRUZ, 9. 2005, Rio de Janeiro. Resumos... Rio de Janeiro: Fundação Oswaldo Cruz, p. 358.

CARDOSO, M. H. W. M.; NÓBREGA, A W.; ABRANTES, S. Efeito da Resposta Cromatográfica Acentuada e Induzida pela Matriz: Estudo de Caso em Tomates. Analytica, n. 34, p. 48-55, abr.-maio 2008.

CODEX ALIMENTARIUS COMISSION. Guidelines on Good Laboratory Practice in Residue Analysis: CAC/GL 40-1993, Rev. 1-2003. Rome: FAO/WHO, 2003. vol. 2A. Disponível em: <http:// www.codexalimentarius.net/download/standards/378/cxg_040e. pdf $>$. Acesso em: 08 out. 2008.

DG-SANCO, EUROPEAN COMISSION. Method Validation and Quality Control Procedures For Pesticide Residues Analysis in Food and Feed. 2007. Document No. SANCO/2007/3131. Disponível em: <http://ec.europa.eu/food/plant/protection/ resources/ qualcontrol_en.pdf $>$. Acesso em: 6 set. 2008.

EURACHEM. The fitness for purpose of analytical methods, a laboratory guide to method validation and related topics. Teddington: LGC, 1998.

FAJGELJ, A.; AMBRUS, A. Guidelines for single-laboratory validation of analytical methods for trace-level concentrations of organic chemicals. In: FAJGELJ, A.; AMBRUS, A. (Ed.). Principles and practices of method validation. Cambridge: Royal Society of Chemistry, 2000. cap. X, p. 179-252.

GENERAL INSPECTORATE FOR HEALTH PROTECTION. Analytical Methods for Pesticide Residues in Foodstuffs. $6^{\text {th }}$ ed. The Hague: The Netherlands. Ministery of Public Health, Welfare and Sport, 1996. Part I.

GREEN, J. M. A practical guide to analytical method validation. Analytical Chemistry News \& Features, v. 68, n. 9, p. 305A-309A, 1996.
HAJŠLOVA, J. et al. Matriz-induced effects: a critical point in the gas chromatographic analysis of pesticide residues. Journal of Chromatography A., v. 800, n. 2, p. 283-295, 1998.

HILL, A. R. C.; REYNOLDS, S. L. Guidelines for in-house validation of analytical methods for pesticide residues in food and animal feeds. Analyst, v. 124, n. 6, p. 953-958, 1999.

HUBER, L. Validation of analytical methods: review and strategy. LC/ GC International, p. 96-105, Feb. 1998.

LEE, M. et al. Multipesticide residue method for fruits and vegetables: California Department of Food and Agriculture. Fresenius Journal Analytical Chemistry, v. 339, n. 6, p. 376-383, 1991.

MILLER, J. C.; MILLER, J. N. Statistics for Analytical Chemistry. New York: Ellis Horwood Limited, 1984.

MOREIRA, L. F. Diagnóstico dos problemas ecotoxicológicos causados pelo uso de inseticida (metamidofós) na região agrícola de Viçosa. 1995. 95 f. Dissertação (Mestre em Agroquímica) Universidade Federal de Viçosa, Viçosa,1995.

MORELLI-CARDOSO, M. H. W. et al. Extraction and clean-up method for the determination of twenty organochlorine pesticide residues in tomatoes by GLC-ECD. Journal of High Resolution Chromatography, v. 22, n. 11, p. 619-622, 1999.

NATIONAL ASSOCIATION OF TESTING AUTHORITIES - NATA Format and content of test methods and procedures for validation and verification of chemical test methods. Sidney: NATA, 1997. 8 p. Technical note 17 .

PIMENTEL, M. T.; BARROS NETO, B. Calibração: Uma Revisão para Químicos Analíticos. Química Nova, v. 19, n. 3, p. 268-277, 1996.

RIBANI, M. et al. Validação em métodos Cromatográficos e eletroforéticos. Química Nova, v. 27, n. 5, p. 771-780, 2004.

SILVA, J. M. et al. Agrotóxico e Trabalho: uma combinação perigosa para a saúde do trabalhador rural. Ciência \& Saúde Coletiva, v. 10, n. 4, p. 891, 2005.

THOMPSON, M.; ELLISON, S. L. R.; WOOD, R. Harmonized Guidelines for Single Laboratory Validation of Methods of Analysis (IUPAC Technical Report). Pure and Applied Chemistry, v. 74, n. 5, p. 835-855, 2002.

THOMPSON, M.; ELLISON, S. L. R.; WOOD, R. The International Harmonized Protocol for the Proficiency Testing of Analytical Chemistry Laboratories (IUPAC Technical Report). Pure and Applied Chemistry, v. 78, n. 1, p. 145-196, 2006.

UNITED STATES DEPARTMENT OF AGRICULTURE - USDA. Pesticide Data Program. SOP No: POP-QC-13. Required Compounds, Marker Pesticides, Process Control Compounds and PDP Commodity Groups. 2008. Disponível em: <http://www. ams.usda.gov/AMSv1.0/getfile?dDocName=STELPRDC5059897>. Acesso em: 26 set. 2008.

WOOD, R. How to validate analytical methods. Trends in Analytical Chemistry, v. 18, n. 9-10, p. 624-632, 1999.

ZROSTLÍKOVÁS, J. et al. Performance of programmed temperature vaporizer, pulsed splitless and on-column injection techniques in analysis of pesticide residues in plant matrices. Journal of Chromatography A., v. 937, n. 1-2, p. 73-86, 2001. 